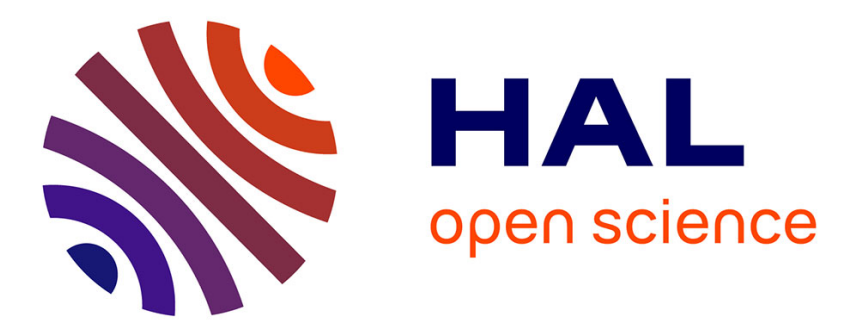

\title{
Générateurs d'impulsions rapides très haute tension à éclateurs
}

F. Schmitt, J. Gresser, G. Metzger, M. Riedinger, G. Sutter

\section{To cite this version:}

F. Schmitt, J. Gresser, G. Metzger, M. Riedinger, G. Sutter. Générateurs d'impulsions rapides très haute tension à éclateurs. Revue de Physique Appliquée, 1969, 4 (2), pp.221-222. 10.1051/rphysap:0196900402022100 . jpa-00243229

\section{HAL Id: jpa-00243229 https://hal.science/jpa-00243229}

Submitted on 1 Jan 1969

HAL is a multi-disciplinary open access archive for the deposit and dissemination of scientific research documents, whether they are published or not. The documents may come from teaching and research institutions in France or abroad, or from public or private research centers.
L'archive ouverte pluridisciplinaire HAL, est destinée au dépôt et à la diffusion de documents scientifiques de niveau recherche, publiés ou non, émanant des établissements d'enseignement et de recherche français ou étrangers, des laboratoires publics ou privés. 


\title{
GÉNÉRATEURS D'IMPULSIONS RAPIDES TRÈS HAUTE TENSION A ÉCLATEURS
}

\author{
F. SGHMITT, J. GRESSER, G. METZGER, M. RIEDINGER et G. SUTTER, \\ Institut de Recherches Nucléaires, Strasbourg.
}

\begin{abstract}
Résumé. - Cet article présente une gamme de générateurs d'impulsions couvrant les tensions allant de $5 \mathrm{kV}$ à $600 \mathrm{kV}$. Les caractéristiques des impulsions obtenues sont : un front de montée très raide et une parfaite reproductibilité. Le délai des générateurs est ramené à des valeurs très faibles avec un jitter négligeable.
\end{abstract}

Abstract. - This paper describes high-voltage pulse generators in the 5 to $600 \mathrm{kV}$ region yielding : fast risetime, small delay, negligible jitter, perfect reproductibility.

I. Introduction. - Les générateurs décrits ici sont destinés à déclencher différents types de chambres à étincelles avec distance interélectrodes variant de quelques millimètres à plusieurs dizaines de centimètres. Ces chambres nécessitent des impulsions rapides d'amplitude comprise entre $5 \mathrm{kV}$ et $600 \mathrm{kV}$ et, pour un fonctionnement correct, le générateur devra notamment satisfaire aux critères suivants $: i$ ) délai très faible entre l'instant de déclenchement du générateur et l'instant où l'impulsion de très haute tension est délivrée (au maximum quelques dizaines de nanosecondes); ii) temps de montée de l'impulsion délivrée très faible (inférieur à $10 \mathrm{~ns}$ ); iii) jitter en temps de l'impulsion de sortie très petit (de l'ordre de la nanoseconde); iv) parfaite reproductibilité des caractéristiques du générateur.

La gamme de générateurs que nous avons conçue comporte un certain nombre de traits communs : a) tous les générateurs fonctionnent sous atmosphère d'azote à des pressions variant entre la pression atmosphérique et 5 bars; $b$ ) l'amplitude des impulsions se règle simplement, d'une part par ajustage de la pression d'azote, d'autre part par ajustage de la tension d'ali-

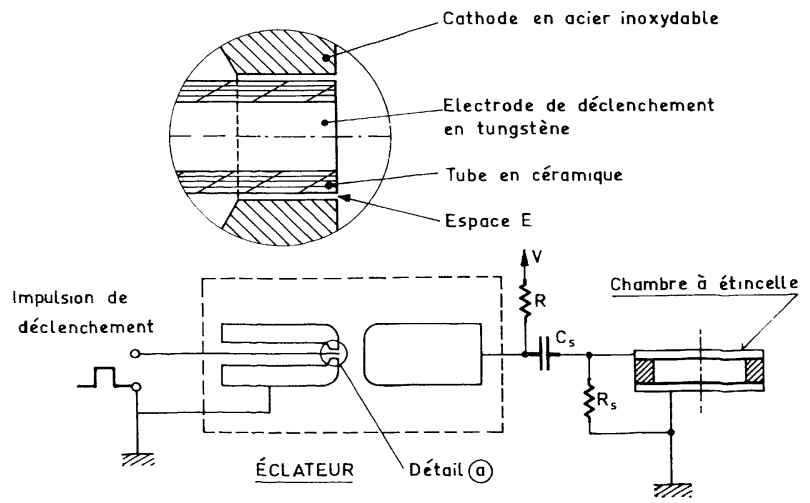

FIG. 1. - Éclateur de déclenchement. mentation continue; c) tous les générateurs utilisent un même type d'électrode de déclenchement. Cette électrode (fig. 1) permet de réduire le délai propre de chaque générateur. Par ailleurs, sa structure permet une grande fiabilité du système de déclenchement et évite le fonctionnement erratique certaines fois rencontré dans les générateurs utilisant un autre mode de déclenchement. Son principe repose sur le fait que le champ électrique existant dans un diélectrique de permittivité élevée est bien plus réduit que celui existant dans un diélectrique de permittivité faible. Ceci permet de retrouver pratiquement toute la tension appliquée entre l'électrode de déclenchement et la cathode, entre cette dernière et l'extérieur du cylindre de céramique délimitant l'espace $\mathbf{E}$ (le cylindre gazeux qui constitue cet espace a une épaisseur de l'ordre de $0,1 \mathrm{~mm}$ ) [1].

II. Générateur à décharge de capacité ( fig. 1). Il permet d'obtenir des impulsions d'amplitude comprise entre 5 et $25 \mathrm{kV}$. Le délai minimal obtenu est de l'ordre de $4 \mathrm{~ns}$, pour une amplitude du signal de déclenchement de $3 \mathrm{kV}$ produit par un multiplicateur de tension du type Marx à 34 étages de transistors [2] fonctionnant en régime d'avalanche. Le temps de montée de l'impulsion délivrée par ce générateur est de 3 ns lorsqu'il est chargé par une résistance non inductive de $50 \Omega$. Le jitter en temps de l'impulsion n'est pas mesurable (Tektronix 519), donc très inférieur à 1 ns.

III. Générateur à éclateur coaxial. - Afin de faire fonctionner des chambres à étincelles à entreplaques comprises entre 5 et $10 \mathrm{~cm}$, nous avons construit un générateur permettant de délivrer des impulsions de forme parfaitement définie. Pour obtenir ce résultat, nous avons intégré l'éclateur dans une ligne coaxiale de $50 \Omega$ d'impédance caractéristique. Cette ligne a le double but d'amener l'impulsion à la chambre à étincelles avec un minimum de perte et de déformation, et de 
permettre la mesure précise de l'impulsion grâce à un atténuateur capacitif incorporé dans la ligne et qui ne perturbe sa structure que très faiblement.

L'amplitude maximale des impulsions délivrées par ce générateur est de l'ordre de $50 \mathrm{kV}$. Comme il faut une tension continue d'alimentation de valeur double, il a été nécessaire, pour des raisons de sécurité, d'entourer les résistances de charge et les capacités de décharge d'une enceinte équipotentielle reliée à la terre. Cette disposition a l'avantage supplémentaire de blinder le générateur et par conséquent de réduire au minimum le rayonnement. Le temps de montée de l'impulsion de sortie est de $5 \mathrm{~ns}$ et le jitter en temps est négligeable lorsqu'on se place très près du claquage autonome.

IV. Générateur du type Marx. - Afin de couvrir la gamme des tensions comprises entre $50 \mathrm{kV}$ et $600 \mathrm{kV}$, nous avons construit un générateur basé sur le principe de la charge en parallèle et de la décharge en série d'un ensemble de 21 capacités, la mise en série se faisant par éclateurs. Ses caractéristiques essentielles sont : i) les courbes d'efficacité présentent des flancs extrêmement raides, l'efficacité croît de 0 à $100 \%$ pour une variation de tension d'alimentation de l'ordre de $100 \mathrm{~V}$; ii) le délai minimal est de $30 \mathrm{~ns}$ et le jitter en temps inférieur à $1 \mathrm{~ns}$. Ceci est essentiellement dû à la réaction interne provoquée par la liaison optique entre éclateurs permettant la production d'électrons par rayons ultraviolets au niveau de la cathode des éclateurs; iii) le temps de montée de l'impulsion de sortie est $4 \mathrm{~ns}$ à vide et $20 \mathrm{~ns}$ lorsque le générateur est chargé par une capacité de 20 pF.
La mesure de l'impulsion de sortie du générateur a présenté de sérieuses difficultés, du fait que le générateur n'était que partiellement blindé. Nous avons cependant obtenu des résultats satisfaisants en utilisant un atténuateur capacitif placé à la sortie du générateur et en effectuant la mesure à une trentaine de mètres du générateur.

V. Circuit de mise en forme pour l'obtention d'impulsions de très faible durée. - Afin de faire fonctionner des chambres à étincelles en « streamer mode ", nous avons adjoint au générateur de Marx un circuit de mise en forme ( fig. 2) avec lequel nous pouvons obtenir des impulsions de durée variable (une dizaine à une centaine de ns) à flancs raides (5 à $10 \mathrm{~ns}$ ) sur une charge de $55 \mathrm{pF}$.

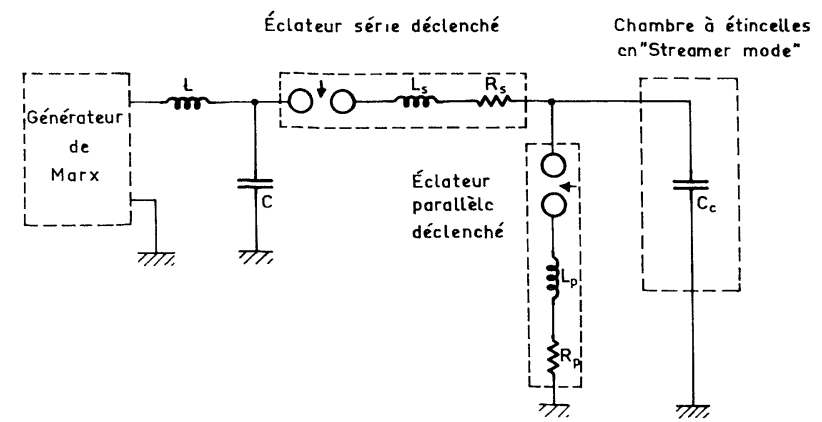

FIG. 2.

Circuit de mise en forme d'une impulsion T.H.T

\section{BIBLIOGRAPHIE}

[1] LAvoIE (L.), Rev. Sci. Instr., 1964, 35, 1567.

[2] Schmit' (F.) et al., Nucl. Instr. Methods, 1967, 52, 331. 